\title{
Parallel-excision infrabrow blepharoplasty with extensive excision of the orbicularis oculi muscle in an Asian population
}

\author{
Yoon Jae Lee ${ }^{1}$, SeongAe $\mathrm{Kim}^{2}$, Jisung Lee ${ }^{3}$, Joong Geel Chung ${ }^{4}$, Young Joon Jun ${ }^{2}$ \\ ${ }^{1}$ Department of Plastic and Reconstructive Surgery, Yeouido St. Mary's Hospital, College of Medicine, The Catholic University of Korea, Seoul; \\ ${ }^{2}$ Department of Plastic and Reconstructive Surgery, Seoul St. Mary's Hospital, College of Medicine, The Catholic University of Korea, Seoul; \\ ${ }_{3}^{3}$ WELL Plastic Surgery Clinic, Seoul; ${ }^{4} J o o n g$ Geel Chung Clinic of Plastic Surgery, Seoul, Korea
}

Background Infrabrow blepharoplasty has become a common surgical method used to rejuvenate aged upper eyelids in Asians. In this paper, we describe the parallel excision method for infrabrow blepharoplasty as a useful alternative to the conventional elliptical excision method. The authors' experience over a 3-year period is presented and reviewed.

Methods A retrospective review of parallel excision infrabrow blepharoplasty cases at our hospital between 2014 and 2017 was performed. Three oculoplastic surgeons compared preoperative and postoperative photographs using the Strasser grading system.

Results From the medical records of 123 patients, a total of 93 patients with moderate-tosevere bilateral dermatochalasis were selected as subjects. The exclusion criterion was levator function less than $8 \mathrm{~mm}$. The total mean follow-up period was 2 years (range, 0.5-3.5 years). The mean skin excision height and width were $9.75 \mathrm{~mm}$ (range, 5-16 mm) and $58.51 \mathrm{~mm}$ (range, 42-75 mm), respectively. All patients who underwent surgery recovered without major complications, and all patients had high levels of satisfaction and improvements in their visual field. In the Strasser evaluation performed by the oculoplastic surgeons, most patients were found to have excellent results.

Conclusions The parallel excision method for infrabrow blepharoplasty is a safe and effective technique that yields more natural- and youthful-looking eyelids than the conventional elliptical excision method. In our method, more effective manipulation of the orbicularis oculi muscle led to a reduction in frontalis compensation, resolution of sunken eyelids, and correction of lateral hooding.

Keywords Eyelids / Blepharoplasty / Eyebrow
Correspondence: Joong Geel Chung Joong Geel Chung Clinic of Plastic Surgery, Eu Lim B.D 2F, 766 Samsungro, Gangnam-gu, Seoul 06070, Korea Fax: +82-2-523-1975

E-mail: jgchung1004@hanmail.net

Young Joon Jun

Department of Plastic and Reconstructive Surgery, Seoul St. Mary's Hospital, College of Medicine, The Catholic University of Korea, 222 Banpo-daero, Seocho-gu, Seoul 06591, Korea

Tel: +82-2-2258-6141

Fax: +82-2-594-7230

E-mail: joony@catholic.ac.kr

\section{INTRODUCTION}

Blepharoplasty has become a popular surgical procedure for rejuvenation in Asia. When dermatochalasis develops and lateral hooding becomes severe with age, effective correction with classical blepharoplasty is challenging unless an incision is made via the lateral canthus. After its introduction by Parkes et al. [1], infrabrow blepharoplasty has become a common surgical method 
used to rejuvenate aged upper eyelids in Asians [2-4]. A variety of methods for infrabrow blepharoplasty have been frequently used. To date, these methods include elliptical excision [5], extended excision [6], and extended upward excision [7-9], as well as surgical methods for the transposition of the orbicularis oculi muscle via fixation to the periosteum $[3,4,8,10]$ or to the galea aponeurotica. We studied the parallel excision method for infrabrow blepharoplasty as an alternative to the conventional elliptical excision method. We found that the parallel excision method allowed for the more effective manipulation of the orbicularis oculi muscle. The results were more natural- and youthful-looking upper eyelids, along with a reduction in frontalis compensation and correction of lateral hooding.

\section{METHODS}

\section{Patients}

After obtaining institutional review board approval (IRB No. SC18RESE0136), we performed retrospective chart review for patients who were treated in our hospital between 2014 and 2017. Clinical data (age, sex, eyebrow position, follow-up duration, and complications) were extracted from these charts. Most patients visited the hospital for visual field defects, lateral skin hooding, intertrigo and frontalis compensation, or cosmetic issues. The inclusion criteria to undergo parallel excision infrabrow blepharoplasty were patients; (1) with good levator function ( $8 \mathrm{~mm}$ or greater), (2) with moderate-to-severe dermatochalasis, (3) who raised their eyebrows excessively (frontalis compensation), and (4) who desired aesthetically beautiful eyes. The exclusion criterion was relatively weak levator function (less than $8 \mathrm{~mm}$ ). The surgical results and aesthetic improvement were evaluated using the Strasser grading system as well as the patients' subjective impressions of the results (excellent, good, fair, or insufficient) [11].

All patients enrolled in the study were informed about the procedure and its risks, and all patients provided written informed consent.

\section{Surgical technique}

\section{Preoperative preparation and excision design}

Prior to surgery, surgical planning was conducted with the patient in the sitting position. The upper incision was marked along the inferior margin of the eyebrow, and the lower incision was marked parallel to the upper incision. Medially and laterally, the superior incision was tapered to meet the inferior incision. The additional area of medial skin tapering was designed to extend approximately 5-6 $\mathrm{mm}$ from the head of the eyebrow, and the tapering of the lateral skin incision was designed to extend approximately 7-8 $\mathrm{mm}$ from the tail of the eyebrow. In most cases, the incision was a straight line measuring 50-60 mm (Fig. 1).

\section{Operative technique}

Local anesthesia was administered while the patient was in a supine position on the operating table; $2 \%$ lidocaine and 1 : 200,000 epinephrine were injected using a 30-G needle, followed by waiting for approximately 5 minutes so that complete hemostasis could be attained. Excision was conducted in the medial-to-lateral direction using a \#15 scalpel blade. A trichophytic upper incision was used to minimize scarring through preservation of the hair follicles. A beveled incision approximately $2 \mathrm{~mm}$ in length was made at a $30^{\circ}$ angle. Next, an excision 5-6 cm in width and approximately $10 \mathrm{~mm}$ in height was performed on the lower margin en bloc, including the skin, subcutaneous layer, and orbicularis oculi muscle. The extended excision of the medial and lateral vertical portions of the orbicularis oculi muscle was made to extend 5-6 mm medially and 7-8 $\mathrm{mm}$ laterally for additional excision of the muscle and to prevent formation of a dog-ear deformity (Fig. 1). The orbicularis oculi muscle could then be fully excised horizontally under the skin flap. Next, the upper and lower flaps of the orbicularis oculi muscle were sutured using 6-0 Vicryl sutures (Ethicon, Somerville, NJ, USA) (Fig. 2). Using this procedure, immediate cor-

\section{Fig. 1. Design of parallel excision infrabrow blepharoplasty}

Yellow line: height of parallel excision skin-muscle flap (about 10 $\mathrm{mm}$, parallel). Red line: medially and laterally, the incision was tapered to meet the inferior incision. The medial tapered area of the skin-muscle flap extended 5-6 mm medially from the head of the eyebrow, while the lateral tapered area of the skin-muscle flap extended 7-8 $\mathrm{mm}$ laterally from the tail of the eyebrow. Blue line (dotted): the medial tapered area of additional muscle excision extended 10-12 mm medially from the head of the eyebrow, while the lateral tapered area of additional muscle excision extended 14$16 \mathrm{~mm}$ laterally from the tail of the eyebrow.

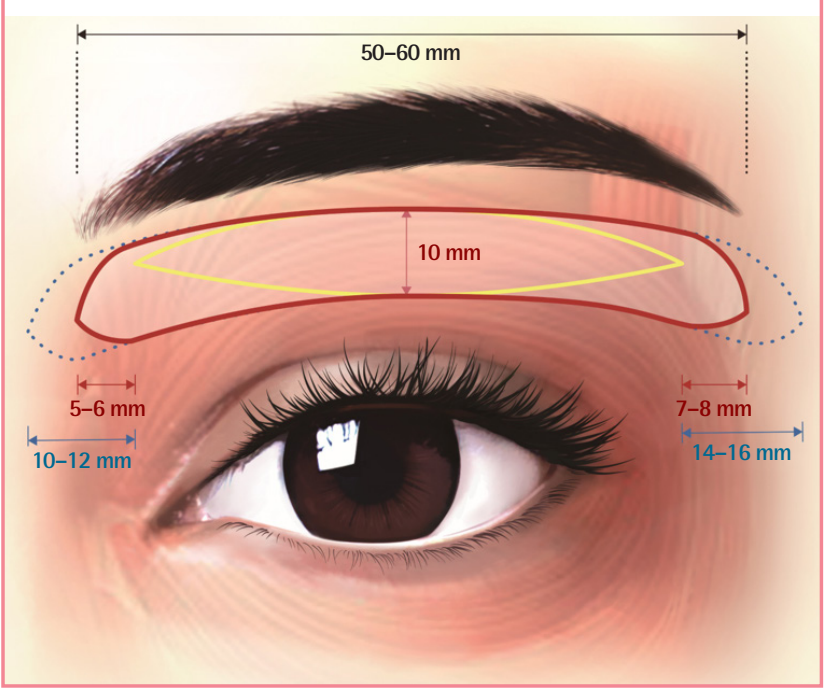


rection of the upper eyelid could be seen with gliding of the lower incisional margin of the skin and orbicularis oculi muscle upward. In addition, the sunken eyelids of some patients were corrected simultaneously by either including orbital fat or moving the sagging fat upward by tucking the orbital septum. The skin was closed using 6-0 black silk continuous sutures (Ethicon). The sutures were removed on postoperative days 4 to 5 (Fig. 2). In cases of dog ear formation, a triamcinolone injection was administered.

\section{RESULTS}

From the medical records of 123 patients (246 eyelids), a total of 93 patients ( 186 eyelids) with moderate-to-severe bilateral dermatochalasis between May 2012 and July 2017 were selected. The subjects included 19 men and 74 women (ratio of male to female, approximately 1:4). The mean age of the patients was
53.9 years, with a range of $21-75$ years. The total mean followup period was 2 years (range, $0.5-3.5$ years). The mean skin excision height and width were $9.75 \mathrm{~mm}$ (range, 5-16 mm) and $58.51 \mathrm{~mm}$ (range, $42-75 \mathrm{~mm}$ ), respectively. All patients who underwent surgery recovered without major complications; one patient underwent a minor revision. Postoperative redness and swelling disappeared quickly, and cases with dog ear formation were resolved using triamcinolone injection. After the sutures were removed 4-5 days postoperatively, the patients were able to continue with their daily lives by applying simple eyebrow makeup or wearing sunglasses. After 3 months, the scars became inconspicuous due to regrowth of the eyebrows. All patients also reported high levels of satisfaction with eyelid function, since the patients experienced ease in eye opening and improvements in their visual fields. Additional details of postoperative changes after infrabrow blepharoplasty using parallel excision are shown in Table 1.

\section{Fig. 2. Parallel excision infrabrow blepharoplasty technique}

(A) Patient with central sunken hollowness and minimal-to-moderate lateral skin redundancy of the upper lid. (B) Preoperative marking for infrabrow blepharoplasty with parallel excision design. (C) Four-month postoperative view shows resolution of dermatochalasis and a more youthful appearance. The skin redundancy, lateral hooding, and central sunken hollowness improved. The vertical pupillary fissure height also increased. (D) The skin and orbicularis muscle flap were excised, exposing the orbital septum. (E) The upper and lower flaps of the orbicularis oculi muscle were sutured using 6-0 Vicryl sutures. (F) The skin was closed using 6-0 black silk sutures.

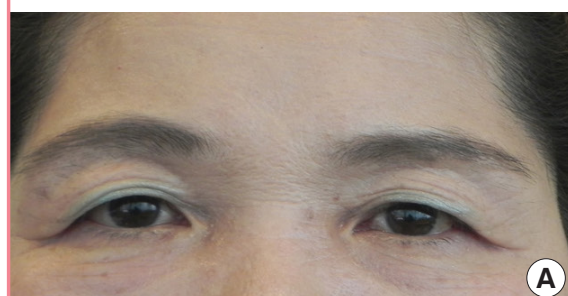

(A)

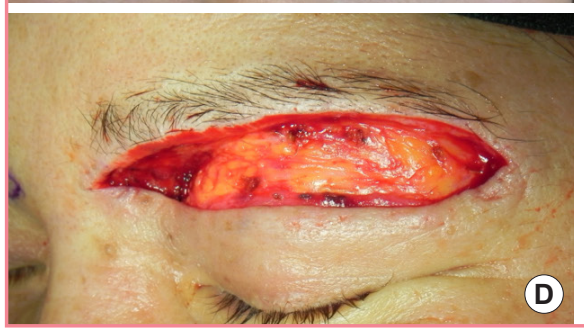

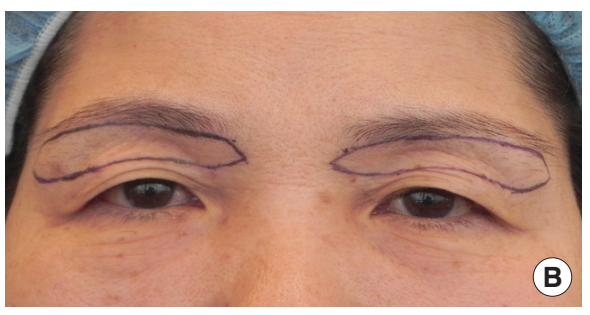
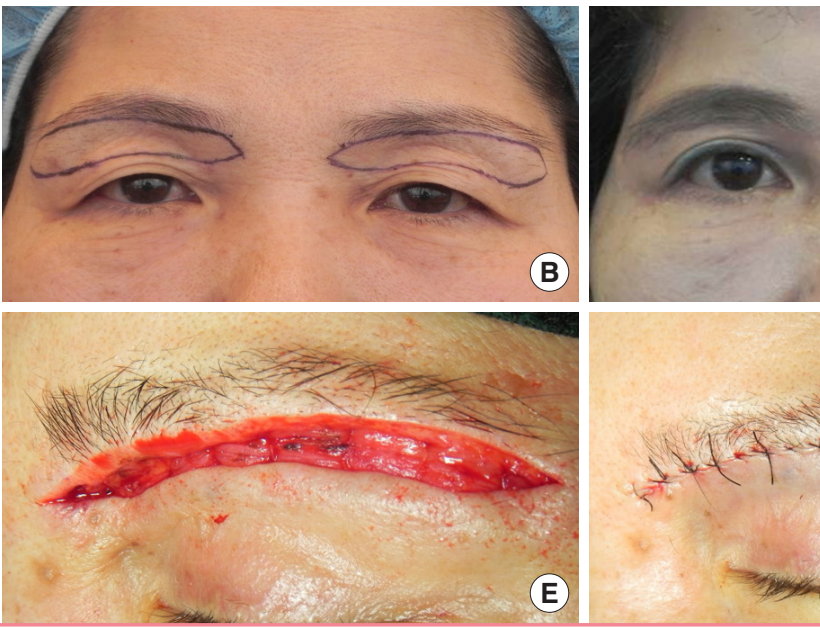

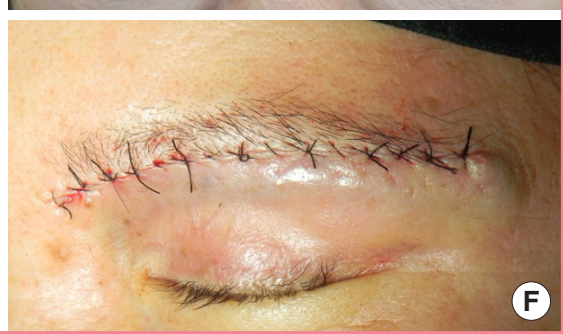

Table 1. Postoperative changes after parallel excision IBB

\section{Before parallel-excision IBB \\ 1. Strong frontalis muscle compensation \\ - Considerable number of transverse frontalis wrinkles \\ - Elevated and asymmetrical eyebrow level (mostly in the dominant eye) \\ - Infrabrow sunken eyelids \\ - Relatively higher lids \\ - Head tilting \\ 2. Small orbital fissure height \\ 3. Excessive lateral hooding with dermatochalasis}

IBB, infrabrow blepharoplasty.

\section{After parallel-excision IBB}

1. Reduction of frontalis muscle compensation

- Decrease or disappearance of forehead transverse wrinkles

- Symmetrical eyebrow level in position

- Correction of sunken eyelids

- Decreased lid height

- Correct head tilting

2. Increase in orbital fissure height

3. Improvement of lateral hooding and dermatochalasis 
Although some patients reported transient numbness around the medial eyebrow, this improved gradually and was followed by sensations of tingling or itching. These sensations gradually disappeared approximately 1 month after surgery for most patients, but took 3 months for three patients.

The objective Strasser grading system for the evaluation of surgical results was applied and the patients' subjective impressions of their aesthetic appearance were collected at 6 months postoperatively. All patients had scores between 0 and 4 points (Table 2), indicating good results. Additionally, $92.5 \%$ of the patients were satisfied with the cosmetic outcome and improvement in dermatochalasis after surgery, as evidenced by results rated excellent or good. Of the 15 patients whose results were rated fair or insufficient, only three rated their level of satisfaction as insufficient; these ratings were due to the formation of dog ears, which vanished with either one or three administrations of triamcinolone injection (1:50 dilution) with a month

Table 2. Summary scores of surgical results according to the objective Strasser grading system

\begin{tabular}{|lcccc|}
\hline $\begin{array}{l}\text { Strasser flaw } \\
\text { category }\end{array}$ & 0 Point & 1 Point & 5 Points & 15 Points \\
\hline Malposition & 93 & 0 & 0 & 0 \\
Distortion & 92 & 1 & 0 & 0 \\
Asymmetry & 93 & 0 & 0 & 0 \\
Contour deformity & 93 & 0 & 0 & 0 \\
Scar & 90 & 3 & 0 & 0 \\
\hline $\begin{array}{l}\text { Values are presented as number. } \\
\text { a) Scores for surgical results according to the Strasser flaw category: perfect, 0 } \\
\text { point; noticeable, 1 point; obvious, 5 points; deforming, 15 points. }\end{array}$ \\
\hline
\end{tabular}

between injections.

Representative preoperative and postoperative photographs are shown in Fig. 3.

\section{DISCUSSION}

We retrospectively reviewed a total of 93 patients (186 eyelids) with moderate-to-severe bilateral dermatochalasis who underwent parallel excision infrabrow blepharoplasty with manipulation of the orbicularis oculi muscle. The total mean follow-up period was 2 years (range, $0.5-3.5$ years). The mean skin excision height and width were $9.75 \mathrm{~mm}$ (range, $5-16 \mathrm{~mm}$ ) and $58.51 \mathrm{~mm}$ (range, $42-75 \mathrm{~mm}$ ), respectively. All patients who underwent surgery recovered without major complications.

The eyes of Asian individuals tend to have small horizontal and vertical pupillary fissures with lateral puffiness and weak double folds $[12,13]$. In addition to the above features, $70 \%$ of patients have frontalis muscle compensation. The greater the ptosis of the eyelid, the stronger the action of the frontalis muscle to compensate. In Asians, another characteristic of the eyes is the presence of more pretarsal and suborbicularis fat than in Caucasians; thus, the distance between the eyebrows and eyelids is longer in Asians than in Caucasians, corresponding to a higher position of the eyebrow [14-17]. With aging, as the inferior projection of fat progresses, the distance between the eyelids and eyebrows increases further, resulting in marked blepharoptosis with dermatochalasis $[18,19]$.

Previously, Lee and Law [5] and Ichinose et al. [2] excised only the skin layer while preserving the orbicularis oculi muscle,

Fig. 3. Parallel excision infrabrow blepharoplasty

(A) A 41-year-old woman complained of a high eyebrow and ptotic upper lid with supratarsal hollowness and lateral hooding. Non-incisional double fold and infrabrow techniques were performed concomitantly. (B) Eight-month postoperative view shows improvement in eyebrow position and supratarsal hollowness. The vertical pupillary fissure height had also increased. (C) A 63-year-old woman with a lateral brow elevation and ptotic upper lid with moderate skin redundancy and lateral hooding. (D) A postoperative view at 3 years and 11 months shows an excellent result regarding eyebrow position and skin redundancy.
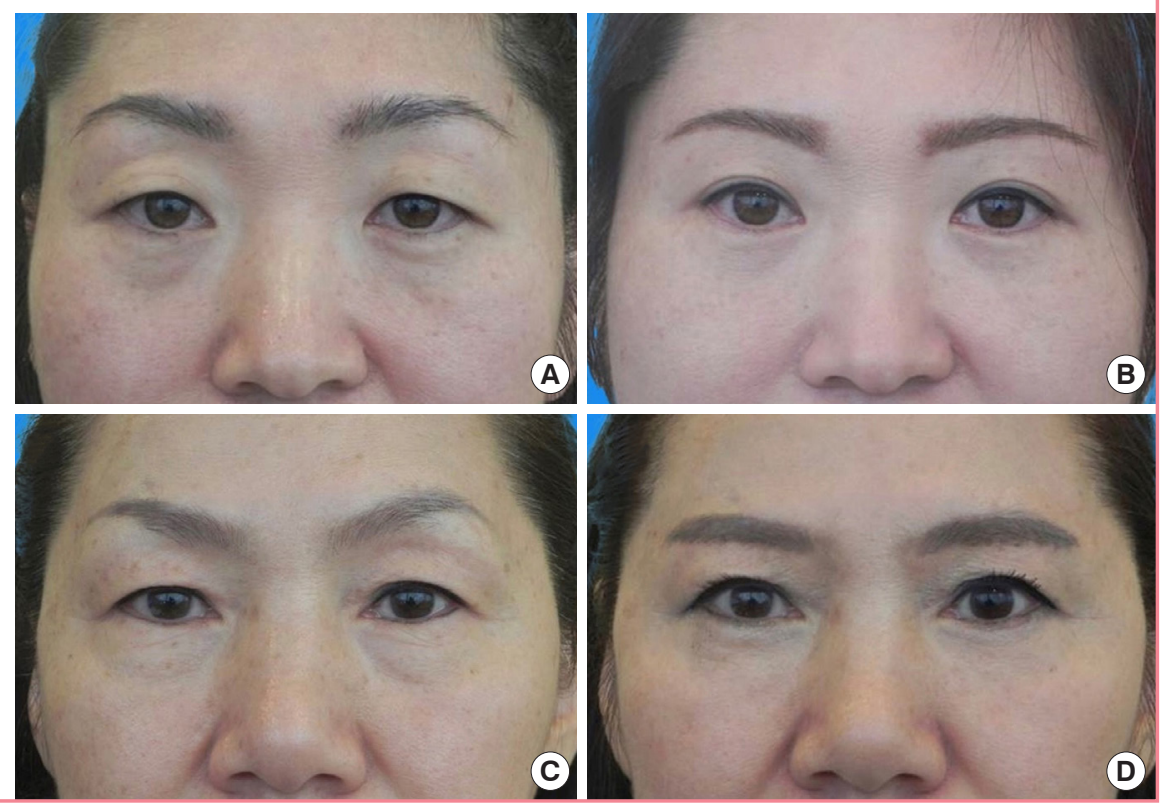
whereas Kim et al. [3] performed en bloc excision, including both the muscles and the skin. Although Sugamata and Yoshizawa [9] performed muscle excision, this excision was only 1-2 $\mathrm{mm}$ deeper than the skin excision. Lee and Hwang [7] divided the depressor muscle by splitting the orbicularis oculi muscle.

In our study, we excised the orbicularis oculi muscle more extensively in parallel than could be excised with an elliptical incision; as such, the medial and lateral vertical portions of the orbicularis oculi muscles were fully removed. This approach enabled (1) reduction of the depression, or downward-pulling, forces exerted by the orbicularis oculi muscles; (2) reduction of weight of the eyelid itself; and (3) the clearance of the obstacle posed by the tissue (orbicularis oculi muscle) while gliding superiorly, resulting in easier eye opening [8].

In excision of the orbicularis oculi, the lower end of the muscle is easily moved upward using sutures in the upper ends of the muscle. The superficially-seated eyelid tissues (skin, orbicularis oculi muscle, and fat) on the same gliding plane are also easily moved upward. These characteristics make the lid structures tension-free during eye opening and closing, which spontaneously allows for dynamic elevation of the upper eyelid and restores the distance between the eyelid and eyebrow so that it resembles that seen at a younger age $[8,9]$. Postoperatively, the eyes have a more natural and youthful appearance, which is long-lasting. In addition, during manipulation of the orbicularis oculi muscle, the sagging contours of the submuscular, pretarsal, and septal fat are corrected by fat tucking that leads to immediate correction of sunken eyelids and translation of the vector in the superior direction [10].
The depression force was also reduced through the shortening of the medial and lateral vertical parts of the orbicularis oculi muscle. Here, "shortening" refers to suturing the end of the mobile lower orbicularis oculi muscle to the end of the immobile upper orbicularis oculi muscle after performing a parallel excision about $1 \mathrm{~cm}$ in height at the medial and lateral vertical aspects of the orbicularis oculi, which presumably exert the greatest depression forces [20-23]. These methods cause an apparent reduction in the depression force exerted by the orbicularis oculi muscle, resulting in easier opening of the eyes and a lower probability of relapse as the orbicularis oculi muscle tone increases with aging [23]. This is because in addition to the two muscles used for elevation (the frontalis and levator palpebrae), the muscles used for depression-the orbicularis oculi-are recruited during eye opening [23].

In every case of upper eyelid surgery, such as the double fold operation, ptosis correction, or infrabrow blepharoplasty, we focused on the manipulation of the orbicularis oculi muscle, not only the excision of the skin. Three main advantages of the parallel design over the elliptical incision method are: reduction in frontalis compensation, correction of sunken eyelids, and improvement of lateral hooding.

In conventional infrabrow approaches, either a strip excision or an elliptical excision is utilized for the orbicularis oculi muscle $[5,19,24]$, or fixation to the periosteum, frontalis, or galea aponeurotica is performed after elliptical excision $[3,4,8,10,25]$. These methods involve insufficient excision of the medial and lateral vertical portions of the orbicularis oculi muscle, causing greater medial and lateral depression forces. The central portion

\section{Fig. 4. Elliptical excision infrabrow blepharoplasty}

(A) Preoperative view of a 49-year-old woman. Infrabrow blepharoplasty was performed with an elliptical design. (B) A postoperative view at 2 years and 2 months shows depression of the central portion of the lid. Elliptical excision leads to a depression and a concave upper lid, especially in the central portion, resulting in cosmetically unfavorable outcomes. (C) Preoperative view of a 63-year-old woman. Infrabrow blepharoplasty of elliptical excision was performed. (D) A postoperative view at 1 year and 4 months shows minimal correction or relapse of lateral hooding.
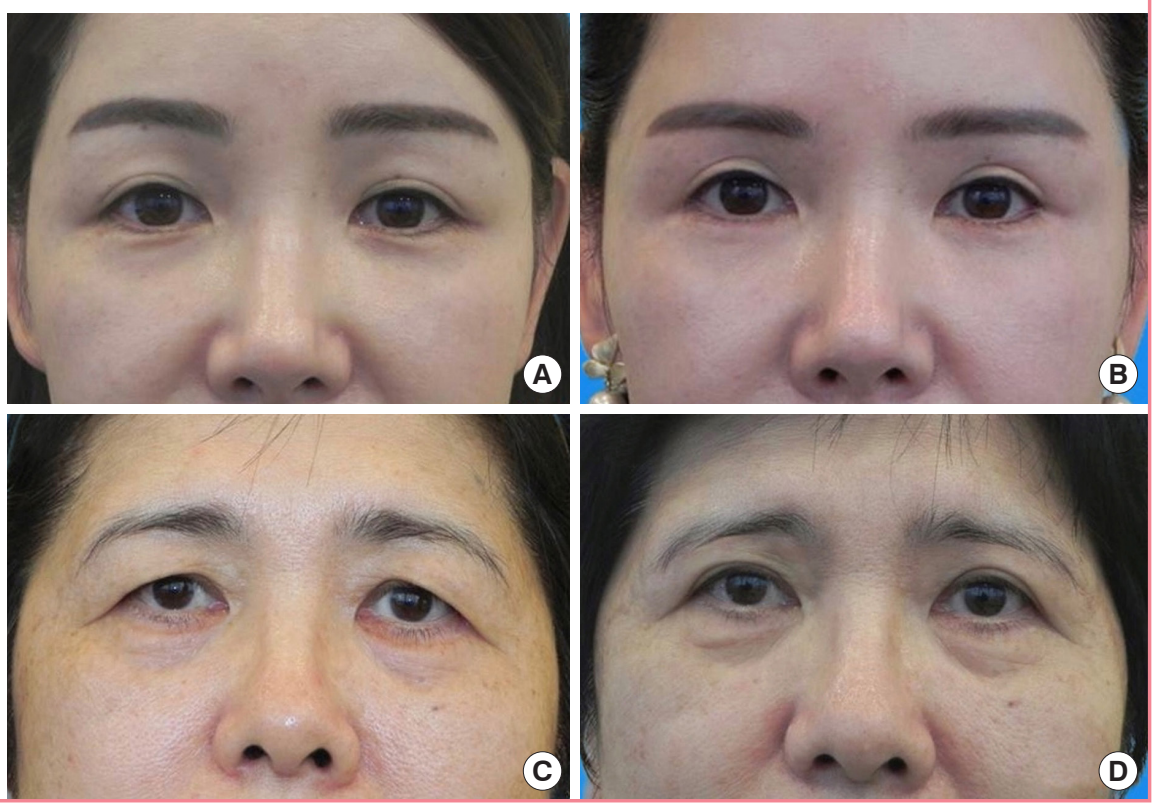
is excised more extensively than the peripheral portion, which results in unnatural upward stretching of the upper eyelid and the formation of a concave surface marked by a depression (Fig. 4). In conventional infrabrow blepharoplasty cases, inadequate excision of the orbicularis oculi muscle results in incomplete correction of frontalis compensation that leads to a sunken eyelid and transverse forehead wrinkles. In such cases, unnecessary additional procedures, such as fat grafting and forehead lifting, are performed. The conventional methods also tend to be associated with a relapse of lateral hooding due to the persistent downward depression force exerted by the orbicularis oculi muscle.

This study had no major limitations. We observed only a few cases of transient numbness in the upper periorbital area. Abnormal sensations usually began a few weeks after surgery, but could occur as early as a few days after the procedure and gradually disappeared within a few months. Careful preservation of the supraorbital nerve and supratrochlear nerve branches reduces the development of these sensations. The surgeon should inform the patient that irritation is a good sign, as it is indicative of the restoration of normal sensation.

Parallel excision infrabrow blepharoplasty is a good option for middle-aged and older Asian women with moderate-to-severe dermatochalasis or frontalis compensation; however, it can also be used in male and young female Asians. This modality can also meet the expectations of patients with aesthetic concerns. For most patients, we now recommend parallel excision infrabrow blepharoplasty as a first choice, unless the patient rejects infrabrow scarring or eyebrow-to-eyelid height reduction.

The parallel excision method of infrabrow blepharoplasty is a reproducible, safe, and effective technique to yield more naturaland youthful-looking eyelids. Through manipulation of the orbicularis oculi muscle, this technique accomplishes a reduction in frontalis compensation, the resolution of sunken eyelids, and the correction of lateral hooding.

\section{NOTES}

\section{Conflict of interest}

No potential conflict of interest relevant to this article was reported.

\section{Ethical approval}

The study was approved by the Institutional Review Board, Catholic Medical Center Office of Human Research Protection Program (IRB No. SC18RESE0136) and performed in accordance with the principles of the Declaration of Helsinki. Written informed consents were obtained.

\section{Patient consent}

The patients provided written informed consent for the publication and the use of their images.

\section{Author contribution}

Conceptualization: Chung JG, Jun YJ. Data curation: Lee YJ, Lee J. Formal analysis: Chung JG, Jun YJ. Methodology: Kim SA, Chung JG. Project administration: Chung JG, Jun YJ. Visualization: Kim SA. Writing - original draft: Lee YJ. Writing - review \& editing: Lee YJ, Chung JG, Jun YJ. Approval of final manuscript: all authors.

\section{ORCID}

Yoon Jae Lee https://orcid.org/0000-0002-8376-9135 SeongAe Kim https://orcid.org/0000-0002-4457-8416

Jisung Lee Joong Geel Chung https://orcid.org/0000-0001-9742-7489 Young Joon Jun https://orcid.org/0000-0001-6537-2460

\section{REFERENCES}

1. Parkes ML, Kamer FM, Merrin ML. Infrabrow lift. Laryngoscope 1976;86:1869-72.

2. Ichinose A, Sugimoto T, Sugimoto I, et al. Extended infrabrow excision blepharoplasty for dermatochalasis in Asians. Arch Facial Plast Surg 2011;13:327-31.

3. Kim YS, Roh TS, Yoo WM, et al. Infrabrow excision blepharoplasty: applications and outcomes in upper blepharoplasty in Asian women. Plast Reconstr Surg 2008; 122:1199-205.

4. Park DH, Baik BS, Nahai F, et al. Cosmetic and reconstructive oculoplastic surgery. 3rd ed. Seoul: Koonja; 2009.

5. Lee D, Law V. Subbrow blepharoplasty for upper eyelid rejuvenation in Asians. Aesthet Surg J 2009;29:284-8.

6. Har-Shai Y, Hirshowitz B. Extended upper blepharoplasty for lateral hooding of the upper eyelid using a scalpel-shaped excision: a 13-year experience. Plast Reconstr Surg 2004; 113:1028-35.

7. Lee EJ, Hwang K. Depressor muscle division through a subbrow excision for the improvement of brow ptosis. J Craniofac Surg 2013;24:1987-90.

8. Fang YH, Liao WC, Ma H. Infraeyebrow blepharoplasty incorporated browpexy in an Asian population. Ann Plast Surg 2013;71 Suppl 1:S20-4.

9. Sugamata A, Yoshizawa N. Infraeyebrow excision blepharoplasty for Japanese blepharochalasis: review of 35 patients over 60 years old. Scand J Plast Reconstr Surg Hand Surg 2010;44:17-20.

10. Kim YS. Subbrow blepharoplasty using supraorbital rim 
periosteal fixation. Aesthetic Plast Surg 2014;38:27-31.

11. Strasser EJ. An objective grading system for the evaluation of cosmetic surgical results. Plast Reconstr Surg 1999;104: 2282-5.

12. Hwang K, Kim DJ, Hwang SH. Thickness of Korean upper eyelid skin at different levels. J Craniofac Surg 2006; 17:54-6.

13. Wang J, Su Y, Zhang J, et al. Subbrow blepharoplasty combined with periorbital muscle manipulation for periorbital rejuvenation in Asian Women. Plast Reconstr Surg 2019; 144:760e-769e.

14. Amrith S. Oriental eyelids: anatomical and surgical considerations. Singapore Med J 1991;32:316-8.

15. Starck WJ, Griffin JE Jr, Epker BN. Objective evaluation of the eyelids and eyebrows after blepharoplasty. J Oral Maxillofac Surg 1996;54:297-302.

16. Gunter JP, Antrobus SD. Aesthetic analysis of the eyebrows. Plast Reconstr Surg 1997;99:1808-16.

17. Kunjur J, Sabesan T, Ilankovan V. Anthropometric analysis of eyebrows and eyelids: an inter-racial study. $\mathrm{Br} \mathrm{J}$ Oral Maxillofac Surg 2006;44:89-93.

18. Yuzuriha S, Matsuo K, Kushima H. An anatomical structure which results in puffiness of the upper eyelid and a narrow palpebral fissure in the Mongoloid eye. Br J Plast Surg 2000; 53:466-72.

19. Kim JH, Lee IJ, Park MC, et al. Aesthetic blepharoptosis correction with release of fibrous web bands between the levator aponeurosis and orbital fat. J Craniofac Surg 2012;23: e52-5.

20. Shadfar S, Perkins SW. Surgical treatment of the brow and upper eyelid. Facial Plast Surg Clin North Am 2015;23:16783.

21. Hahn S, Holds JB, Couch SM. Upper lid blepharoplasty. Facial Plast Surg Clin North Am 2016;24:119-27.

22. Shu M, He L, Su Y, et al. A novel supra-brow combined with infra-brow lift approach for Asian Women. Aesthetic Plast Surg 2016;40:343-8.

23. Burroughs JR, Bearden WH, Anderson RL, et al. Internal brow elevation at blepharoplasty. Arch Facial Plast Surg 2006;8:36-41.

24. Leatherbarrow B. Oculoplastic surgery. 2nd ed. New York: Informa Healthcare; 2011.

25. Yun S, Son D, Yeo H, et al. Changes of eyebrow muscle activity with aging: functional analysis revealed by electromyography. Plast Reconstr Surg 2014;133:455e-463e. 